\title{
Experiences of Parents Caring for Infants with Rare Scalp Mass as Identified through a Disease-Specific Blog
}

\author{
Miranda Worthen, MPhil, PhD, Tara H. Leonard, MPH, Thomas R. Blair, MD, MS, \\ and Nalin Gupta, MD, PhD
}

Background: Delayed subaponeurotic fluid collection (DSFC) is a self-limited disorder of unknown etiology characterized by a benign, fluid-filled mass in the subaponeurotic layer of an infant's scalp. While a few case series describe DSFC, the experiences of families whose infants develop this condition have not previously been reported.

Methods: We used a disease-specific blog to evaluate the experiences of 69 families affected by DSFC. We identified self-reported clinical features of DSFC and qualitatively analyzed the families' experiences with obtaining a diagnosis and care for their infants.

Results: Infants presented in several clinical settings, and multiple diagnostic procedures were administered, including ultrasound (46\%), computed tomography (30\%), and head radiography (22\%). Qualitative themes emerged: lack of provider awareness of DSFC, concern about potentially harmful diagnostic procedures, suspicion of child abuse, and the importance of the website in providing support to families.

Conclusions: Though DSFC can be diagnosed clinically and its natural history is benign, its presence can be emotionally draining for parents. Physicians should be aware of this clinical entity to rapidly allay parental distress and avoid unnecessary procedures. Disease-specific blogs can help providers learn about rare diseases, contain useful clinical information for research, and can benefit patient care by providing social support for families. (J Am Board Fam Med 2015;28:750-758.)

Keywords: Infant Development, Parenting, Qualitative Research, Scalp, Social Media

This study examines the experiences of parents interacting with the medical community for a particular category of infant scalp mass known as delayed subaponeurotic fluid collection (DSFC). While the most common types of neonatal scalp masses, cephalohematoma and caput succedaneum, arise during birth, DSFC typically occur between 5 and 9 weeks of age. Infants with DSFC, therefore, typ-

This article was externally peer reviewed.

Submitted 24 February 2015; revised 21 May 2015; accepted 29 May 2015.

From the Department of Health Science and Recreation, San Jose State University, San Jose, CA (MW, THL); the Semel Institute for Neuroscience and Human Behavior, University of California Los Angeles, Los Angeles (TRB); and the Departments of Neurological Surgery and Pediatrics, University of California San Francisco, San Francisco (NG).

Funding: none.

Conflict of interest: none declared.

Corresponding author: Miranda Worthen, MPhil, PhD, Department of Health Science and Recreation, San Jose State University, One Washington Square, San Jose, CA 95192 (E-mail: miranda.worthen@sjsu.edu). ically present to family physicians, pediatricians, or emergency departments. Providers are often unfamiliar with this rare condition and may never have previously encountered a case. While DSFCs resolve within a few months and are not associated with any adverse sequelae, existing studies suggest that because of a lack of provider awareness of these masses, they frequently lead to unnecessary tests and anxiety for parents and providers alike.

Scalp masses in infants have many etiologies and may arise from various layers of the scalp. From superficial to deep, the 5 major layers of the scalp are skin; a thin layer of relatively loose, subcutaneous tissue; the galea aponeurotica, a fibrous anchor for the previous 2 layers; a second layer of loose connective tissue; and, last, the periosteum, a connective tissue membrane that is attached to the calvarium.

Two well-known causes of scalp masses in infants are cephalohematoma, in which a hematoma is trapped between the periosteum and the skull, 
and the more superficial caput succedaneum, in which a hematoma is trapped between the skin and the galea aponeurotica. Because a cephalohematoma lies below the periosteum, which is rigidly attached to the calvarium, the collection does not cross the suture lines that separate the individual bones that comprise the infant calvarium. The blood or fluid in caput succedaneum, on the other hand, sits above the deep fibrous layers of the scalp and is therefore able to move freely across suture lines. Another site for neonatal bleeding in the scalp is the subgaleal or subaponeurotic space between the galea aponeurotica and the periosteum; neonatal bleeding in this location can become profuse, leading to an obstetric emergency. However, the appearance of masses in this tissue plane weeks after birth, a DSFC, represents a condition that is benign and poorly described in the medical literature.

Aside from developmental anomalies, neonatal scalp masses are usually caused by birth trauma, particularly in the context of deliveries assisted by the use of forceps or vacuum extraction. ${ }^{1-4}$ Vacuum extraction specifically has been found to be associated with cephalohematoma compared with forceps-assisted delivery, ${ }^{5,6}$ but it is not associated with long-term neurologic sequelae. ${ }^{7}$ One large case series that used skull radiography and ultrasonography to examine neonates delivered by vacuum assistance found that cephalohematomas occurred in $10.8 \%$ of patients. ${ }^{8}$ Another large population-based study found an overall occurrence of $1.6 \%$ among neonates. ${ }^{9}$ Subgaleal hematomas are reported to occur following vacuum-assisted delivery. ${ }^{10,11}$ Although generally considered harmless, fetal scalp electrodes are associated with cerebrospinal fluid leak ${ }^{12-15}$ and with local infection. ${ }^{16,17}$ Although rare, the differential diagnosis does include entities such as tumors, pseudocysts, and encephaloceles, which should be considered by the treating provider. Head injuries caused by accident or child abuse must also be considered.

Typically, DSFCs arise between 4 to 18 weeks of age, resolve within a few months, and do not cause developmental delay. Particularly given their delayed timing, DSFCs do not fit with the common clinical patterns observed with cephalohematoma, caput succedaneum, or perinatal subgaleal bleeds, and the relevant literature is limited. In a singlepatient case study, Aries et $\mathrm{al}^{18}$ described a 10 week-old with 2 weeks of subaponeurotic swelling, which was identified as "a result of birth trauma." The authors contrasted the potential risk associated with subgaleal hematomas, which occur at birth, in the same location with the delayed and "benign course" of DSFCs. In an earlier study of 5 cases, Schoberer et $\mathrm{al}^{19}$ stated that DSFC "may be frequently related to traumatic labor and occur within weeks after birth." These authors acknowledged that the "etiology has remained uncertain" but noted that analysis of fluid aspirated from these collections suggested leakage of cerebrospinal fluid. In a series of 6 infants, Hopkins et $\mathrm{al}^{20}$ noted that 4 of 6 patients had vacuum-assisted delivery. These patients presented with "nontender, soft, mobile and fluctuant scalp swellings," all of which resolved within 24 weeks, with "no long-term sequelae." Vaibhav et $\mathrm{al}^{21}$ presented 4 additional cases and reiterated the points noted in earlier publications: the entity is little-known, has a mean age at appearance of 7 weeks and mean age at resolution of 10 weeks, can be diagnosed clinically, and has no detrimental implications for subsequent development.

Recent research has identified social media websites created by and for patients as potentially useful tools for studying rare pediatric diseases. ${ }^{22}$ Because rare diseases usually do not have central databases to record clinical information or treatment results, we made use of disease-specific social media for this study. Using a publicly accessible blog, we assembled a database of cases of DSFC, identifying clinical and diagnostic features based on parental report of 69 case infants. In addition, through close reading of the blog posts, we identified major parental concerns, including the lack of provider awareness of DSFC, worries about diagnostic procedures, and anger when providers suspected child abuse. Parents also reported relief in finding the blog and learning about other infants with the same condition. The aim of this study is to define this clinical entity in terms of both parental experiences and patterns of clinical presentation.

\section{Methods}

\section{Website Identification and Data Collection}

We began by examining the blog entries on a patient-support website called eHealthforum (http:// ehealthforum.com). The specific entries appeared under a blog heading titled, "Fluid Between Scalp And Skull," within a forum for parents of newborn babies. The blog is among the top hits when typing 
the following terms into an Internet search engine: fluid, lump, baby, and head. Only a few of the participants who posted on this blog posted on other blogs within eHealthforum, and many noted that they had located the blog while searching the Internet to understand their infant's condition. The earliest blog entry was from 2005, and the most recent was from 2014. We collected data directly from these public blog postings and analyzed the posts thematically to ascertain clinical patterns, medical responses, and parental experiences. ${ }^{23}$ When sufficient data were available in the posting, the following inclusion criteria were applied: onset between 4 and 18 weeks of age, resolution by 6 months of age, lack of obvious history of trauma, and location of the fluid collection on the scalp.

\section{Online Survey}

We used the blog to recruit participants to complete an online survey about their experiences. After obtaining permission from the website host, we contacted participants via the website's internal E-mail service, inviting them to take part in further study. We had a low response rate to the direct E-mails, likely because blog respondents must "opt in" to being notified of messages waiting in the internal system and therefore may not have received our messages. Surveys were conducted with 4 respondents who consented to participate using Qualtrics software. We reviewed the medical records of 3 of these infants after parents' disclosure of these records. Data presented here, therefore, survey parental experiences of presumed DSFC and aggregate data from labor and delivery as selfreported by families posting on the blog and responding to the survey.

\section{Data Analysis}

Cases that met the inclusion criteria were analyzed by 2 authors (MW and THL), who read the posts to identify qualitative themes using the approach described by Braun and Clarke. ${ }^{23}$ Qualitative themes were developed inductively based on close reading and rereading of the blog posts. Quantitative measures were developed a priori based on review of the extant literature regarding DSFCs. Both MW and THL qualitatively and quantitatively coded several posts, and any discrepancies were discussed and reconciled. THL conducted subsequent coding. The institutional review boards of University of California, San Francisco; University of Califor- nia, Berkeley; University of California, Los Angeles; and San Jose State University approved study procedures.

\section{Results}

\section{Self-Reported Clinical Features}

There were a total of 121 posts, from which we identified 94 unique cases; 69 of these cases met the inclusion criteria for DSFC and were analyzed in this study. This constitutes a convenience sample of infants with DSFC. In addition, 4 parents of case infants completed an online survey, and 3 of these families supplied medical records for review. The data for this study thus include information about 69 case infants: blog posts about all 69 infants, follow-up surveys about 4 of the 69 infants described in the blog, and medical records of 3 of the 4 infants whose parents responded to the survey.

Of the 69 cases, the blog posts or survey identified the age at onset of the DSFC in 68 instances (Figure 1). The mean age at onset was 8.75 weeks. Age at resolution of the mass was noted in 11 cases; the mean was 16.3 weeks. The time from onset of the DSFC to resolution in the 11 cases for which these data were available ranged from 4 to 16 weeks, with a mean time to resolution of 9.5 weeks.

Most cases presented in multiple settings, including the family physician or pediatrician's office (75\%), emergency departments (38\%), and/or a specialists' office (35\%) (Table 1). Cases were likely to experience 1 or more diagnostic procedure, including head radiography, ultrasound, computed tomography (CT), and magnetic resonance imaging (MRI) (Table 2). The most common diagnostic procedure was ultrasound (46\%), followed by CT $(30 \%)$ and head radiography (22\%). Many infants were administered more than 1 diagnostic procedure.

Blog posters noted labor and delivery experiences in approximately half of all cases. In 34 of the 69 cases (49\%), a vacuum was used during labor and delivery, whereas forceps were used in 5 cases (7\%). In 29 cases (42\%), the infant was delivered by cesarean delivery. In addition, fetal monitoring using fetal scalp electrodes was noted in 5 cases (7\%). However, many posts described a "difficult" labor but did not provide details on the labor or delivery experience. Thus the proportions noted above are likely to be underestimates of the actual proportion of cases with each of these labor and delivery experiences. 
Figure 1. Age of onset in weeks among cases with delayed subaponeurotic fluid collection (DSFC) $(n=68)$. Note that cases with onset before 4 weeks or after 18 weeks were not included in our sample because they did not meet the criteria of DSFC. The age at onset was not identified for one case infant.

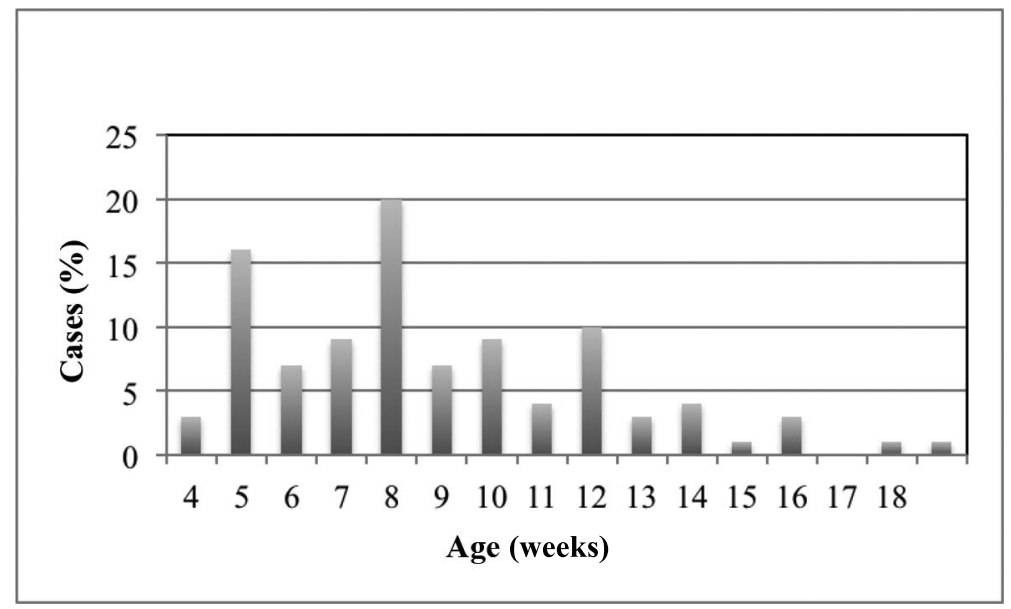

\section{Qualitative Findings}

Four main themes emerged from our qualitative analysis of the blog entries and survey responses: lack of provider awareness of DSFC, concern about the use of unnecessary and potentially harmful diagnostic procedures on patients presenting with the hallmarks of DSFC, suspicion of child abuse, and the critical role of the patient-support website in providing information and emotional support to families. Throughout the posts, parents expressed a high level of anxiety, initially in response to their infants' symptoms and then compounded by their interactions with the medical establishment.

\section{Lack of Provider Awareness}

All the blog participants reported that medical providers were unfamiliar with the clinical presentation of DSFC. Most families initiated treatment

Table 1. Settings in Which Patients Presented

\begin{tabular}{lc}
\hline Setting & Patients Presenting (n) \\
\hline Primary physician's office* & 52 \\
Emergency department $_{\text {Specialist's office }}^{\dagger}$ & 26 \\
Unknown $_{\text {Total }}$ & 24 \\
\hline
\end{tabular}

Many patients presented in more than one location.

*Primary physicians included pediatricians and family practitioners.

${ }^{\dagger}$ Specialists included pediatric neurologists, pediatric neurosurgeons, and pediatric dermatologists. through a family physician, pediatrician, or emergency department doctor. A third of cases were referred to 1 or more specialists, including neurologists and pediatric surgeons, causing delays in diagnosis and continued anxiety. Bloggers used words such as baffled, stumped, puzzled, and perplexed to describe care provider responses to their infants' symptoms. As a result, many of the infants were examined by multiple providers over a series of days or weeks, often without a conclusive diagnosis.

"We took her to the [emergency department], a pediatrician, a consulting pediatrician, and then 2 other pediatricians in a different practice, who all said, 'never seen that before.' We are waiting to go to a pediatric neurologist for additional guidance.”

"We too saw our pediatrician who claimed he had never seen this before. So we went to a

Table 2. Types of Diagnostic Procedures Done

\begin{tabular}{lcc}
\hline Diagnostic Procedure & $\begin{array}{c}\text { Unique } \\
\text { Cases } \\
(\mathrm{n})\end{array}$ & $\begin{array}{c}\text { Percentage of } \\
\text { Total Cases } \\
(\mathrm{n}=69)\end{array}$ \\
\hline Head radiography & 15 & 22 \\
Body radiography & 1 & 1 \\
Ultrasound & 32 & 46 \\
CT & 21 & 30 \\
MRI & 5 & 7 \\
\hline
\end{tabular}

Many patients received more than one diagnostic procedure. CT, computed tomography; MRI, magnetic resonance imaging. 
big-time pediatric neurosurgeon ... who also said it was extremely odd and scheduled an MRI-but we cannot get on the MRI's calendar for another 4 weeks, causing further stress."

"Her doctor said, 'I will be honest, I've never seen this before.' I was shocked! He has been in practice since I was born! Nonetheless, he called and arranged for us to see a neurosurgeon....We could not get in for 1.5 weeks ... and in that time her bump grew even larger and firmer!"

\section{Concern About Diagnostic Procedures}

Many blog participants listed the number and type of diagnostic procedures performed on their infant. These tests included head radiography, full-body radiography, ultrasound, CT, and MRI. A number of parents expressed concerns about the potential harm such procedures could have on their infant's health. Others shared details about the emotional trauma caused by the process itself.

"I am worried about him having 2 CT scans before he was 9 weeks old.... I am not sure what affects the radiation can have on him. His doctor ordered them and I assumed she would not have done so unless it were entirely necessary."

"The radiograph was so incredibly difficult to go through because we had to hold her down with her arms by her side and she was so scared and confused and crying so hard. It literally brought my husband and I to tears watching her go through it."

"We saw all the 'best' doctors and no one knew what it was. So we went and got a CT scan (which still keeps me up at night) and an MRI. . . Took about 10 years off my life too!”

\section{Suspicion of Child Abuse}

In 9 of the cases (13\%), blog participants described being questioned about their own behavior in relation to their child's symptoms. Both subtle and overt accusations included questions about the infant's environment, supervision, and siblings' behavior.

"He took one look at her, smiled, and said, 'She got whacked in the head.' I said something indignant like 'What do you mean? Of course she did not get whacked in the head!' He replied that yes, indeed, these bumps are caused by a whack or a knock of some sort on the noggin. He said sometimes a sibling hits them when you are not looking."

"What scared us the most was when the neurologist came back and ordered her to have a full body radiograph. ... My wife started crying because the doctors kept asking if we had dropped her or something.... Of course the docs did not find anything after those tests as well."

Three parents who posted on the blog (4\%) were contacted by Social Services to investigate child abuse. As a result, those infants received additional diagnostic procedures.

"The next thing I know, I am told that due to an unexplained head injury, the hospital has contacted social services and my son's entire body has to be [X-rayed] so they can check for fractures!!! Of course [X-rays] show nothing but we are now being investigated by social services to rule out abuse!!! I am furious about the ignorance of the [pediatrician] and other [doctors] which has now lead to a social services investigation of "possible abuse'!!!"

\section{Critical Role of the Patient-Support Website}

Blog participants voiced a range of strong emotions, including fear, anxiety, frustration, and anger. Initial concerns about their infants' symptoms had been compounded, rather than relieved, by interaction with the medical establishment. Many wrote that the patient-support website was their only source of information and support.

"So glad I found this website. ... It seems even though all our doctors have never heard of or seen this before that we have a commonality among us.... I can breathe easier now knowing that our baby is not the only one in the world to experience this."

\section{Discussion}

This study contributes to our current understanding of DSFC by examining a parent-reported cohort of 69 infants as parents sought diagnosis and management of the condition. Case information was obtained from a publicly accessible blog, and 
Table 3. Factors Supporting a Clinical Diagnosis of Delayed Subaponeurotic Fluid Collection

\begin{tabular}{ll}
\hline History & $\begin{array}{c}\text { Appearance between approximately } 4 \text { and } 18 \\
\text { weeks of age }\end{array}$ \\
Absence of reported distress or deficits \\
Use of vacuum extraction in delivery \\
Cesarean delivery \\
Absent indicators for accidental or \\
nonaccidental trauma \\
No apparent distress; no signs of trauma \\
Patient meeting developmental milestones \\
Visible scalp mass without ecchymoses or \\
signs of inflammation \\
No pulsations or skull defects present
\end{tabular}

several key variables reported in other case reports of DSFC were available. The average age at onset of the DSFC in this series ( 8.75 weeks) is very similar to that previously reported $(7.5$ weeks $){ }^{21}$ However, the average age at resolution in our series (16.3 weeks) was quite a bit older compared with the series described by Vaibhav et $\mathrm{al}^{21}$ (10 weeks). Determination of "resolution" may reflect considerable subjectivity among appraising parents or physicians.

The etiology of DSFC remains unknown. Various labor and delivery practices have been implicated as possible causative agents, including the use of assistive measures such as vacuum ${ }^{20}$ and/or scalp electrodes. ${ }^{24}$ Vaibhav et $\mathrm{al}^{21}$ summarized the delivery characteristics of the extant cases in the literature: instrument assistance was used in 10 of 15 cases, and vacuum assistance in 9 of the 15 cases. In our cohort, vacuum extraction was used in approximately $50 \%$ of the cases. It is possible that gradual liquefaction of a subgaleal hematoma may lead to delayed recognition of a birth-related injury. The data we collected do not support or disprove any particular etiology of DSFCs. Further research should be done to determine whether there are common associations with other labor and birth variables, such as duration of labor, fetal position, or use of scalp electrodes.

Case infants described in this blog received care from a wide variety of medical providers, including family physicians, emergency department doctors, pediatricians, and pediatric neurologists. Almost none of the providers consulted by blog posters seem to have been familiar with DSFC. As a result, many infants were seen by several different providers, and parents often went days to weeks without assurance that their babies' scalp masses were benign, causing significant emotional distress.

In addition, because of a lack of awareness of DSFC and standard discriminant diagnostic procedures, many providers recommended multiple diagnostic tests. These tests frequently included radiographs and CT scans, both of which have been associated with an increased risk of cancer when administered during infancy. ${ }^{25-32}$ Parents accessing the blog shared their concern about these diagnostic procedures. Because DSFC is a benign condition, observation is the best plan, and multiple diagnostic tests are unnecessary.

Because of its rarity, many providers never encounter DSFC, and those who do may not recognize this entity. Based on the literature and the clinical features we identified through analysis of this disease-specific blog and infant medical records, we suggest a set of factors to support the clinical diagnosis of DSFC (Table 3). We also provide an image of an infant with DSFC to support recognition of this entity (Figure 2). We concur with Vaibhav et $\mathrm{al}^{21}$ that though DSFC can be diagnosed clinically without the use of radiologic investigation, each case must be evaluated individually and appropriate diagnostic tests should be ordered if there are any concerns. In such instances, providers should consider the possible harms of any test when weighing which test is most appropriate.

Parental distress often was compounded by the lack of a clear diagnosis, multiple visits to different care providers, time delays in receiving diagnostic

Figure 2. Infant with delayed subaponeurotic fluid collection (DSFC). This infant presented with DSFC at 8 weeks. In this photograph, she is 22 weeks old and the mass is resolving.

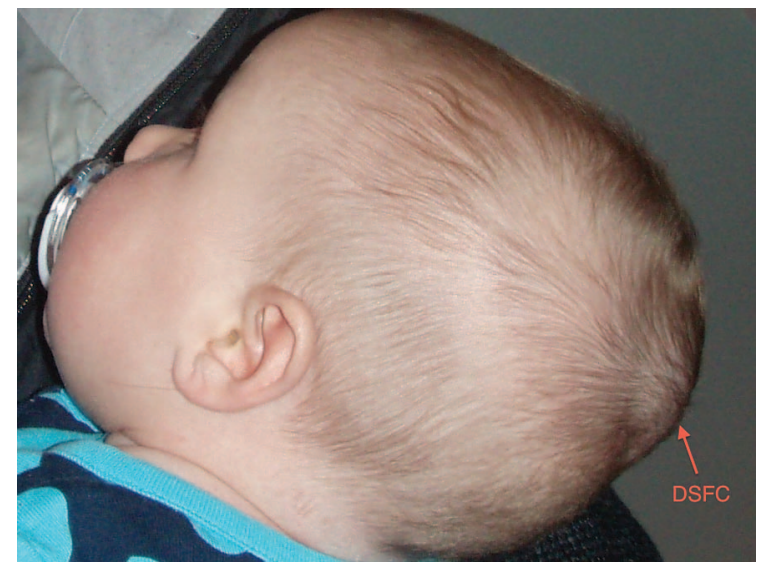


services, and questioning from medical providers that implied parental misconduct. In 3 cases, providers involved Social Services because of suspicion of child abuse. As Vaibhav et $\mathrm{al}^{21}$ suggest, it is reasonable to consider inflicted trauma when evaluating an infant with an unexplained scalp mass. However, knowledge of a benign diagnostic entity such as DSFC may have provided the clinician with an alternative explanation, potentially avoiding unnecessary CT scans, specialist referrals, and involvement of Child Protective Services. Providers have an obligation to screen for abuse, but we recommend that such screening be done cautiously and considerately to avoid causing parental distress and subjecting infants to unnecessary procedures.

Researchers have increasingly used social media to obtain information about medical illnesses. A systematic review of the literature on cancer and social media found that most studies using social media focus on how patients access social support through disease-specific social media. ${ }^{33}$ In this study, parents reported that finding the blog and hearing about other infants with the same condition was relieving, especially in light of their providers' lack of awareness of DSFC. Family physicians and other providers should know that online support groups and disease-specific blogs exist and can be excellent resources for patients and families, especially those struggling with rare diseases.

Social media interventions have also been developed to recruit patients with rare diseases to online studies. $^{22}$ The parents of a child with a previously unknown genetic condition recently used social media to successfully locate other children with the same disorder, catalyzing research to benefit all children with the condition and suggesting a new model of collaboration between parents, providers, and researchers. ${ }^{34}$

In this study we used social media to gather self-reported clinical information about a rare condition, giving us the opportunity to describe 4 times as many cases as have previously been identified in the literature and to assess the parents' experience of seeking diagnosis and care for a child with DSFC. However, relying on parents' report of clinical features of the scalp mass and recollection of clinical encounters and diagnostic procedures has the potential to introduce bias. In the 3 cases for which we reviewed medical records, the records supported the parent's report. As the proliferation of patient-driven social media presents a potentially valuable new data source for studying rare conditions, further research should evaluate the validity of publicly shared descriptions of clinical features and medical encounters.

One limitation of this study is that we do not know the characteristics of the target population of infants with DSFC and thus do not know how representative users of the family support blog are of this total population. We can speculate that families who seek care for their infants with DSFC from family physicians, pediatricians, or emergency departments, and who are quickly given a diagnosis of DSFC and reassured about its benign course, may not go online to find more information about their infant's condition. Similarly, visitors to the blog who decide to post about their own experiences likely represent a subset of all visitors to the blog with infants experiencing similar situations. We do not claim that the blog posts analyzed in this study are representative of all infants with DSFC. However, together with the extant literature on DSFC, these posts do suggest that DSFC remains little known and poorly understood.

While the natural history of DSFC is benign, its presence can be emotionally draining for parents. Also, infants are exposed to excess radiation from unnecessary imaging, leading to increased risk of cancer. With further description and better education of clinicians and parents alike, DSFC may become an entity that is easily diagnosed and managed.

This study suggests that disease-specific blogs can be a source of clinical information for researchers seeking to learn more about rare diseases. $\mathrm{Al}$ though such resources are subject to minimal scrutiny and are therefore a secondary resource for clinical guidance, providers can also learn from these blogs about how to care for patients with rare diseases and which diagnostic procedures are helpful in diagnosing rare conditions. In the case of DSFC, for example, a clinician consulting this blog may decide not to recommend radiography or CT based on the clinical information contained in the posts. In addition, providers should be aware of these resources to refer patients to these blogs for social support from other families affected by rare diseases.

The authors thank Anna Shillinglaw for inspiring this study, Stefan Timmermans for his thoughtful suggestions, and all the blog participants for their contributions. 


\section{References}

1. Demissie K, Rhoads GG, Smulian JC, et al. Operative vaginal delivery and neonatal and infant adverse outcomes: population based retrospective analysis. BMJ 2004;329:24-9.

2. Doumouchtsis SK, Arulkumaran S. Head trauma after instrumental births. Clin Perinatol 2008;35: 69-83.

3. Hughes CA, Harley EH, Milmoe G, Bala R, Martorella A. Birth trauma in the head and neck. Arch Otolaryngol Head Neck Surg 1999;125:193-9.

4. Towner D, Castro MA, Eby-Wilkens E, Gilbert WM. Effect of mode of delivery in nulliparous women on neonatal intracranial injury. N Engl J Med 1999;341:1709-14.

5. Caughey AB, Sandberg PL, Zlatnik MG, Thiet MP, Parer JT, Laros RK Jr. Forceps compared with vacuum: rates of neonatal and maternal morbidity. Obstet Gynecol 2005;106(5 Pt 1):908-12.

6. Johanson RB, Menon BK. Vacuum extraction versus forceps for assisted vaginal delivery. Cochrane Database Syst Rev 2000;(2):CD000224.

7. Ngan HY, Miu P, Ko L, Ma HK. Long-term neurological sequelae following vacuum extractor delivery. Aust N Z J Obstet Gynaecol 1990;30:111-4.

8. Simonson C, Barlow P, Dehennin N, et al. Neonatal complications of vacuum-assisted delivery. Obstet Gynecol 2007;109:626-33.

9. Baskett TF, Allen VM, O'Connell CM, Allen AC. Fetal trauma in term pregnancy. Am J Obstet Gynecol 2007;197:499.e1-7.

10. Reid J. Neonatal subgaleal hemorrhage. Neonatal Netw 2007;26:219-27.

11. Uchil D, Arulkumaran S. Neonatal subgaleal hemorrhage and its relationship to delivery by vacuum extraction. Obstet Gynecol Surv 2003;58:687-93.

12. Nieburg P, Gross SJ. Cerebrospinal fluid leak in a neonate associated with fetal scalp electrode monitoring. Am J Obstet Gynecol 1983;147:839-40.

13. Riskin A, Beni-Adani L, Chistiekov I, Kugelman A, Abend-Weinger M, Bader D. Cerebrospinal fluid leak in a premature neonate. Am J Perinatol 2005; 22:57-9.

14. Sorokin Y, Weintraub Z, Rothschild A, Abramovici H, Iancu TC. Cerebrospinal fluid leak in the neonate-complication of fetal scalp electrode monitoring. Case report and review of the literature. Isr J Med Sci 1990;26:633-5.

15. Turbeville DF, McCaffree MA. Fetal scalp electrode complications: cerebrospinal fluid leak. Obstet Gynecol 1979;54:469-70.

16. Feder HM, Jr., MacLean, Moxon R. Scalp abscess secondary to fetal scalp electrode. J Pediatr 1976;89: 808-9.

17. Listinsky JL, Wood BP, Ekholm SE. Parietal osteomyelitis and epidural abscess: a delayed complication of fetal monitoring. Pediatr Radiol 1986;16:150-1.
18. Aries MJ, van Dam SW, Hoving EW, Brouwer OF. An infant with a cranial swelling: sub-aponeurotic fluid collection. Ned Tijdschr Geneeskd 2009;153: A713.

19. Schoberer A, Yagmur E, Boltshauser E, Korinth M, Niggemann P, Hausler M. Sub-aponeurotic fluid collections: a delayed-onset self-limiting cerebrospinal fluid fistula in young infants. Eur J Paediatr Neurol 2008;12:401-3.

20. Hopkins RE, Inward C, Chambers T, Grier D. Subaponeurotic fluid collections in infancy. Clin Radiol 2002;57:114-6.

21. Vaibhav A, Smith R, Millman G, Cooper J, Dwyer J. Subaponeurotic or subgaleal fluid collections in infancy: an unusual but distinct cause of scalp swelling in infancy. BMJ Case Rep 2010;2010. pii: bcr0420102915.

22. Schumacher KR, Stringer KA, Donohue JE, et al. Social media methods for studying rare diseases. Pediatrics 2014:133:e1345-53.

23. Braun V, Clarke V. Using thematic analysis in psychology. Qual Res Psychology 2006;3:77-101.

24. Petraglia AL, Moravan MJ, Marky AH, Silberstein HJ. Delayed sub-aponeurotic fluid collections in infancy: three cases and a review of the literature. Surg Neurol Int 2010;1. pii:34.

25. Bartley K, Metayer C, Selvin S, Ducore J, Buffler P. Diagnostic X-rays and risk of childhood leukaemia. Int J Epidemiol 2010;39:1628-37.

26. Krille L, Zeeb H, Jahnen A, et al. Computed tomographies and cancer risk in children: a literature overview of CT practices, risk estimations and an epidemiologic cohort study proposal. Radiat Environ Biophys 2012;51:103-11.

27. Mathews JD, Forsythe AV, Brady Z, et al. Cancer risk in 680,000 people exposed to computed tomography scans in childhood or adolescence: data linkage study of 11 million Australians. BMJ 2013;346:f2360.

28. Miglioretti DL, Johnson E, Williams A, et al. The use of computed tomography in pediatrics and the associated radiation exposure and estimated cancer risk. JAMA Pediatr 2013;167:700-7.

29. Pearce MS, Salotti JA, Little MP, et al. Radiation exposure from CT scans in childhood and subsequent risk of leukaemia and brain tumours: a retrospective cohort study. Lancet 2012;380:499-505.

30. Shu XO, Potter JD, Linet MS, et al. Diagnostic $\mathrm{X}$-rays and ultrasound exposure and risk of childhood acute lymphoblastic leukemia by immunophenotype. Cancer Epidemiol Biomarkers Prev 2002;11: $177-85$.

31. Smith-Bindman R, Lipson J, Marcus R, et al. Radiation dose associated with common computed tomography examinations and the associated lifetime 
attributable risk of cancer. Arch Intern Med 2009; 169:2078-86.

32. Wakeford R. The risk of childhood leukaemia following exposure to ionising radiation-a review. J Radiol Prot 2013;33:1-25.

33. Koskan A, Klasko L, Davis SN, et al. Use and taxonomy of social media in cancer-related research: a systematic review. Am J Public Health. 2014;104: e20-37.

34. Might M, Wilsey M. The shifting model in clinical diagnostics: how next-generation sequencing and families are altering the way rare diseases are discovered, studied, and treated. Genet Med 2014;16: 736-7. 